\title{
Fermi and Gamow-Teller Strength in Charge Exchange with Radioactive Beams
}

\author{
C. A. Bertulani ${ }^{a}$ and P. Lotti ${ }^{b}$ \\ a Instituto de Física, Universidade Federal \\ do Rio de Janeiro \\ 21945-970 Rio de Janeiro, RJ, Brazil. E-mail: bertu@if.ufrj.br \\ ${ }^{b}$ INFN, Sezione di Padova, via F. Marzolo 8, \\ I-35131 Padova, Italy
}

\begin{abstract}
At forward angles, and bombarding energies $E>200 \mathrm{MeV}$, the $(p, n)$ and $(n, p)$ reactions are thought to be directly proportional to the Gamow-Teller transition strengths in the nuclei. Assuming that this relationship also holds for charge exchange induced by high-energy heavy ions, it would be very useful in studies with radioactive beams. Contrary to this expectation, we show that the determination of Gamow-Teller and Fermi matrix elements from heavy-ion charge-exchange at forward angles is very inaccurate.
\end{abstract}

Typeset using REVTEX 
A major experimental and theoretical effort is under way to study the properties and applications of drip-line nuclei (for a review, see ref. [1]). One of the main issues of this rapidly growing field is to understand the basic features of many unstable nuclei, which can only be assessed by using beams of radioactive nuclei. Of great relevance is the study of spin-isospin properties in unstable nuclei. A well-defined proportionality between heavy ion charge-exchange and beta-decay transition strengths would have important consequences. Such a relationship would be particularly useful to allow beta transition strength functions to be mapped out in drip-line nuclei. An accurate link between heavy ion exchange reactions and the well understood beta-decay processes would also put our understanding of the nuclear structure and reaction mechanisms of unstable nuclei on a surer quantitative footing.

It is well known that $(p, n)$ and $(n, p)$ charge-exchange reactions at intermediate energies $(E>100 \mathrm{MeV})$ have been a powerful spectroscopic tool for measuring Gamow-Teller (GT) transition matrix elements [2,3]. This approach can also be used in reactions with radioactive beams, using a hydrogen gas target. However, due to the low luminosity of the beams, this process yields low counting rates. A more promising procedure would be the use of heavy ion charge exchange (HICEX) instead of $(p, n)$ collisions. In ref. [4] the possibility to use HICEX as a probe of Gamow-Teller matrix elements was suggested and a relation between the forward cross section and the Gamow-Teller matrix elements was found. A possible drawback of this approach is the complication originated by the internal structure of the target nucleus. This method was used as a guidance in ref. [5] to draw conclusions on Gamow-Teller (and Fermi) matrix elements in ${ }^{13} \mathrm{C}$ and ${ }^{13} \mathrm{~N}$ from the mirror nuclei reaction ${ }^{13} C\left({ }^{13} N,{ }^{13} C\right){ }^{13} N$.

In this article we develop a strong absorption model of HICEX based on a single particle approach. We use this simple structure model for qualitative purposes only. Our aim is to access the reliability of the relationship of HICEX measurements and the GamowTeller and Fermi matrix elements. The key point to be discussed here is that if we cannot show the existence of a valid relationship for such a simple model and for rather simple reaction partners, then very probably it would not exist for more complicated nuclear models. Because of the use of mirror nuclei, and their isospin symmetry, the experimental data on the ${ }^{13} C\left({ }^{13} N,{ }^{13} C\right){ }^{13} N$ reaction is also an ideal test of the theory.

In heavy-ion charge exchange reactions it is important to know the underlying nature of the exchange mechanism. In ref. [6] it was shown that in heavy ion collisions at high energies $(E>100 \mathrm{MeV}$.A) the exchange mechanism is dominated by the fundamental $\pi$ and $\rho$ - exchange. The differential cross section for the process is given by 


$$
\frac{d \sigma}{d \Omega}=\frac{k^{\prime}}{k}\left(\frac{\mu}{4 \pi^{2} \hbar^{2}}\right)^{2}\left(2 J_{1}+1\right)^{-1}\left(2 J_{2}+1\right)^{-1} \sum_{\alpha_{1}, \alpha_{2}}\left|T_{i f}\left(\alpha_{1}, \alpha_{2}\right)\right|^{2}
$$

where $\mu$ is the reduced mass of the system $(1+2), k\left(k^{\prime}\right)$ is the initial (final) center-of-mass momentum, $J_{1}$ and $J_{2}$ are the angular momenta in the entrance channel, and the sum is an average over initial angular momentum projections and a sum over final angular momentum projections, denoted respectively by $\alpha_{1}$ and $\alpha_{2}$.

The transition matrix $T_{i f}$ is given by [7]

$$
\left.T_{i f}=\frac{1}{(2 \pi)^{3}} \int d^{3} R \chi^{(-) *}(\mathbf{R}) \chi^{(+)}(\mathbf{R})\right\} \int d^{3} q e^{i \mathbf{q} \cdot \mathbf{R}} \mathcal{M}\left(\alpha_{1}, \alpha_{2}, \mathbf{q}\right)
$$

where $\mathbf{R} \equiv(\mathbf{b}, z)$ is the relative coordinate between the nuclei, $\chi^{(-)}(\mathbf{R})\left[\chi^{(+)}(\mathbf{R})\right]$ is the incoming (outgoing) scattering wave obtained in terms of the optical potential for elastic scattering, and

$$
\mathcal{M}=\int d^{2} r_{1} d^{3} r_{2} \delta \rho_{1}\left(\mathbf{r}_{\mathbf{1}}\right) \delta \rho_{2}\left(\mathbf{r}_{\mathbf{2}}\right) e^{i \mathbf{q} \cdot\left(\mathbf{r}_{1}-\mathbf{r}_{2}\right)} V_{e x c h}(\mathbf{q})
$$

where $\delta \rho_{i}$ is the transition density of the nucleus $i$, which includes spin, isospin and spatial transitions. The meson-exchange potential $V_{O B E P}$ is a sum of $\pi-, \rho-$, and $\omega$-exchange interaction. We use a modified meson-exchange interaction to include a non-spin-flip term: $V_{\text {exch }}=V_{\text {OBEP }}+V_{\tau} \tau_{1} \cdot \tau_{2}$ [7,9].

The eq. 0.3 is separable in terms of the spin and isospin variables, yielding $\mathcal{M}=$ $\mathcal{M}_{12}+\mathcal{M}_{2 \leftrightarrow 1}$, where

$$
\begin{aligned}
\mathcal{M}_{12} & =\int d^{2} q\left\{F_{\sigma \tau}(\mathbf{q}) \sum_{\mu=1}^{3}<\alpha_{f}(1)\left|\tau_{1}^{(+)} \sigma_{\mu} e^{i \mathbf{q} \cdot \mathbf{r}_{1}}\right| \alpha_{i}(1)><\alpha_{f}(2)\left|\tau_{2}^{(-)} \sigma_{\mu} e^{i \mathbf{q} \cdot \mathbf{r}_{2}}\right| \alpha_{i}(2)>\right. \\
& +G_{\sigma \tau}(\mathbf{q}) \sum_{\mu, \mu^{\prime}=1}^{3} \hat{q}_{\mu} \hat{q}_{\mu^{\prime}}<\alpha_{f}(1)\left|\tau_{1}^{(+)} \sigma_{\mu} e^{i \mathbf{q} \cdot \mathbf{r}_{1}}\right| \alpha_{i}(1)><\alpha_{f}(2)\left|\tau_{2}^{(-)} \sigma_{\mu^{\prime}} e^{i \mathbf{q} \cdot \mathbf{r}_{\mathbf{2}}}\right| \alpha_{i}(2)> \\
& +H_{\tau}(\mathbf{q})<\alpha_{f}(1)\left|\tau_{1}^{(+)} e^{i \mathbf{q} \cdot \mathbf{r}_{1}}\right| \alpha_{i}(1)><\alpha_{f}(2)\left|\tau_{2}^{(-)} e^{i \mathbf{q} \cdot \mathbf{r}_{2}}\right| \alpha_{i}(2)>
\end{aligned}
$$

In the equation above the sums over $\mu$ and $\mu^{\prime}$ run over the spherical components of the spin vector. $F_{\sigma \tau}(\mathbf{q}), G_{\sigma \tau}(\mathbf{q})$ and $H_{\tau}(\mathbf{q})$ are functions of the central $(\mathrm{L}=0)$ and the tensorial $(\mathrm{L}=2)$ component of the charge-exchange potential.

Since the initial and final wavefunctions are decomposed into their spatial and spindependent parts, it is appropriate to expand the exponential functions in eq. 0.4 into multipoles and use the Wigner-Eckart theorem, to obtain sums over products of Clebsh-Gordan coefficients and reduced matrix elements of the form $<\phi_{j^{\prime}}^{(f)}\left\|j_{I}(q r)\left[\sigma \otimes Y_{I}\right]_{I^{\prime}} \tau^{( \pm)}\right\| \phi_{j}^{(i)}>$, $<\phi_{j^{\prime}}^{(f)}\left\|j_{I}(q r)\left[\sigma \otimes Y_{I}\right]_{I^{\prime}}\right\| \phi_{j}^{(i)}>$, and $<\phi_{j^{\prime}}^{(f)}\left\|j_{I}(q r) \tau^{( \pm)}\right\| \phi_{j}^{(i)}>$. These matrix elements can be 
calculated using eqs. (A.2.19) and (A.2.24) of ref. [8]. They involve geometric coefficients and overlap integrals of the form

$$
\mathcal{I}_{I j j^{\prime}}(q)=\int R_{j^{\prime}}(r) R_{j}(r) j_{I}(q r) r^{2} d r,
$$

in terms of the single-particle wavefunctions $R_{j}(r)$. The cross section results from interference of terms corresponding to several possibilities of angular momentum transfer.

From 0.4 it is straightforward to show the relationship between the heavy-ion chargeexchange cross sections and the Gamow-Teller and Fermi matrix elements. In beta-decay experiments a similar matrix element appears, but with the exponential factors replaced by $\exp i\left(\mathbf{p}_{e}+\mathbf{p}_{\nu}\right) . \mathbf{r}$, where $\mathbf{p}_{e}$ and $\mathbf{p}_{\nu}$ are the electron and the neutrino momentum, respectively. This exponential factor can be approximated by the unity, if $|\mathbf{r}|<\hbar / p_{e}$ and $\hbar / p_{\nu}$. Since the energies of the light particles involved in beta-decay are of the order of a few $\mathrm{MeV}$, the Compton wavelengths of the electron and the neutrino are approximately equal to $10^{3} \mathrm{fm}$, i.e., about hundred times larger than the nuclear sizes over which the integration occurs. This contrasts with charge exchange experiments, where the exponential factor in 0.4 involves the Compton wavelength of $\pi$ and $\rho$. Thus, the relation q.r $\ll 1$ is not well justified for HICEX.

Assuming that the "low-q" (or long-wavelength) approximation is also valid for HICEX, i.e., inserting eq. 0.4, with the exponentials replaced by the unity in eqs. 0.1-0.4, one gets

$$
\frac{d \sigma}{d \Omega} \simeq c_{1} B_{1}(G T) B_{2}(G T)+c_{2} B_{1}(F) B_{2}(F)+c_{3}\left[B_{1}(F) B_{2}(F) B_{1}(G T) B_{2}(G T)\right]^{1 / 2},
$$

where

$B(G T)=\frac{1}{2 j+1} \sum_{\alpha^{\prime}, \alpha}\left|\left\langle\alpha^{\prime}\left|\sigma \tau^{( \pm)}\right| \alpha\right\rangle\right|^{2}$ and $B(F)=\frac{1}{2 j+1} \sum_{\alpha^{\prime}, \alpha}\left|\left\langle\alpha^{\prime}\left|\tau^{( \pm)}\right| \alpha\right\rangle\right|^{2}$

are the Gamow-Teller and Fermi strengths, respectively. The coefficients $c_{1}, c_{2}$, and $c_{3}$ are products of geometric factors and integrals over the $q$ - and $R$-coordinates. The "low$q "$ approximation 0.6 also includes a sum over all single-particle transitions, which is not explicitly shown for simplicity.

Eq. 0.6 is the result that one would like to have. Assuming that the charge-exchange potential (corrected for medium effects, etc.) is accurately known and that the same is true for the scattering waves, then the coefficients $c_{i}$ could be reliably calculated. An experimental measurement of HICEX would then provide the magnitude of $B(G T)$ 's and $B(F)$ 's for several nuclei. This would be a specially important technique for studies of dripline nuclei. We now investigate if this relation is valid using the ${ }^{13} C\left({ }^{13} N,{ }^{13} C\right){ }^{13} \mathrm{~N}$ reaction at 105 $\mathrm{MeV} /$ nucleon [5] for comparison. 
Following ref. [9] we normalize the interaction so that at zero momentum transfer $V_{O B E P} \equiv V_{\sigma \tau}=265 \mathrm{MeV} \cdot \mathrm{fm}^{3}$ and $V_{\tau}=100 \mathrm{MeV} \cdot \mathrm{fm}^{3}$, appropriate for proton laboratory energies of order of $100 \mathrm{MeV}$. We describe the scattering waves in terms of eikonal wavefunctions. Since an optical potential for this reaction at $100 \mathrm{MeV} /$ nucleon is not known, we use an optical potential which fits the reaction ${ }^{12} C+{ }^{12} C$ at $85 \mathrm{MeV} /$ nucleon [11, [1]. To calculate the single-particle wavefunctions we use a potential of the form $V=V_{0} f(r)+V_{S O}(\mathbf{l} . \sigma)(d f / d r) / r$, with $f(r)=\left[1+\exp \left(r-R_{0}\right) / a\right]^{-1}$, for the neutrons. For the protons we add the Coulomb potential of a uniformly charged sphere of radius $R_{0}$. For both ${ }^{13} \mathrm{C}$ and ${ }^{13} \mathrm{~N}$ we choose the set of parameters $V_{0}=-53 \mathrm{MeV}, R_{0}=2.86 \mathrm{fm}, a=0.65 \mathrm{fm}$, and $V_{S O}=-15.5 \mathrm{MeV}$. In this simple model, the relevant transitions are $1 p_{1 / 2}^{\pi} \longrightarrow 1 p_{1 / 2}^{\pi^{\prime}}(\pi=n$, or $p$, i.e., one proton in ${ }^{13} \mathrm{~N}$ goes to a neutron in ${ }^{13} \mathrm{C}$, and vice-versa) involving the operator $\tau$ (Fermi transitions). For Gamow-Teller transitions ( $\sigma \tau$ operator) the relevant transitions are $1 p_{1 / 2}^{\pi} \longrightarrow 1 p_{1 / 2}^{\pi^{\prime}}$ and $1 p_{1 / 2}^{\pi} \longrightarrow 1 p_{3 / 2}^{\pi^{\prime}}$.

In table 1 we show the matrix elements of the single particle transitions: $B_{1 / 2^{-}} \equiv$ $B_{1 / 2^{-}} \longrightarrow B_{1 / 2^{-}}$and $B_{3 / 2^{-}} \equiv B_{1 / 2^{-}} \longrightarrow B_{3 / 2^{-}}$for Fermi and Gamow-Teller transitions. For comparison, we also give the values of these matrix elements, in the case that the overlap integral includes a spherical Bessel function of zeroth-order, $j_{0}(q r)$, for additional two values of $q$. The matrix elements are drastically reduced for large values of $q$, especially for $1 p_{1 / 2} \longrightarrow 1 p_{3 / 2}$ transitions.

In figure 1 we compare the experimental [5] and the theoretical calculation of the differential charge exchange cross section for the double excitation case, i.e. when both target $\left({ }^{13} \mathrm{C}\right)$ and projectile $\left({ }^{13} N\right)$ go to the $3 / 2^{-}$state. Both experimental data and the calculation are given in arbitrary units. Thus, only the angular dependence of the cross section can be discussed. The angular dependence is rather insensitive to the structure model for the nuclei, but strongly sensitive on the parameters of the optical potential used to calculate the scattering wavefunctions. Only when such parameters are known from, e.g., elastic scattering one can have a better knowledge of the influence of the Gamow-Teller and Fermi matrix elements on the charge exchange cross section. This, of course, assuming that the exchange potential is well known. In fact, the form of the exchange potential is crucial for this aim.

In figure 2 we plot the ratio between the exact calculation and the low-q approximation, eq. 0.6. With the single particle basis the expansion can be stopped at the $I=5$ term. In fact, the term with $I=0$ dominates. This is shown in the figure where the contribution of the $I=0$ (dashed curve) term of the expansion is shown. The total sum includes terms up to $I=5$ (solid curve). However, note that the ratio between the exact calculation and the low-q approximation amounts to a factor of 5 at the forward direction. This difference can be 
understood in a simple way by using eq. 0.2. If strong absorption and final state interactions could be neglected, $\chi^{(-) *}(\mathbf{R}) \chi^{(+)}(\mathbf{R}) \approx \exp \{i \mathbf{Q} \cdot \mathbf{R}\}$, where $\mathbf{Q}=\mathbf{k}^{\prime}-\mathbf{k}$ is the momentum transfer to the c.m. scattering. Thus, the integral over $\mathbf{R}$ yields a delta function, $\delta(\mathbf{q}-\mathbf{Q})$. That is, $Q=0$ (forward scattering) would imply $q=0$, too. Very forward scattering would then justify replacing the exponentials in eq. 0.4 by unity, and an equation of the form of eq. 0.6 would be obtained. However, strong absorption modifies this scenario: $\mathbf{Q}$ is not directly related to $\mathbf{q}$, and the equation 0.6 is a bad approximation. The larger the absorption radius, the worse this approximation should be. This may somewhat explain why for $(p, n)$ reactions the low-q approximation is better justified, since the absorption radius is smaller in that case. But, even for $(p, n)$ reactions the low-q approximation seems to be ill-defined, as was shown recently [12], especially for the weak transitions.

Another possibility to get direct information on the Fermi and Gamow-Teller strengths from HICEX reactions would be in the case that the expansion of the exponential function in 0.4 can be stopped at the term with $I=0$. One gets matrix elements of the form

$$
<\phi_{j^{\prime} l^{\prime} m^{\prime}}^{\pi^{\prime}}\left|\sigma_{\mu} \tau^{\nu} j_{0}(q r)\right| \phi_{j l m}^{\pi}>=\hat{j}\left(j 1 m \mu \mid j^{\prime} m^{\prime}\right) \mathcal{F}_{j j^{\prime}}(q)[B(G T)]^{1 / 2}
$$

and a similar expression for Fermi transitions. The function $\mathcal{F}(q)_{j j^{\prime}}=\left|\mathcal{I}_{0 j j^{\prime}}(q) / \mathcal{I}_{0 j j^{\prime}}(q=0)\right|^{2}$ has to be calculated with accuracy in this case. Similar arguments are given in ref. [3] for $(p, n)$ reactions.

In figure 3 we show the percentage deviation between the exact calculation and a calculation done with only the $I=0$ term of the expansion. Also shown (dashed curve) is the differential cross section in arbitrary units. At $\theta=0$ the difference is about $2 \%$ and increases largely close to the minima of the cross section. This is because, close to the minima (which are basically determined by the $I_{0}$ term) the spherical Bessel functions $j_{I>0}$ contribute more to the integrals.

Although the $(I=0)$-approximation is not bad at $\theta=0$ compared to the full calculation, it cannot be considered as a useful tool to obtain the Fermi and Gamow-Teller strengths from HICEX, unless a good model for the overlap integrals can be done. This is however just what one would like to extract from the experiments, and not from theory. As one sees from table 1 the matrix elements involved in HICEX are strongly modified by the short range property of the exchange interaction. Only a small part of the transition densities, close to the surface of the nucleus is relevant for the matrix elements. In contrast, in beta-decay the whole transition density is relevant. To show this we decrease artificially the masses of the pion and the rho by a factor $\alpha$. These masses enter into the form of the exchange interaction and are responsible for its range [9,7]. As we see from figure 4, as $\alpha$ increases, 
increasing the range of the interaction, the ratio between the exact calculation and the low-q approximation decreases, reaching unity at $\alpha \approx 10$. This means that eq. 0.6 would be valid for an interaction of range $r_{0} \approx 10 \times\left(\hbar / m_{\pi} c\right) \approx 10 \mathrm{fm}$.

In conclusion, we have shown that it is very difficult to obtain reliable estimates of Gamow-Teller or of Fermi strength from HICEX experiments. This might be surprising, since weak interaction strengths determined from $(p, n)$ reactions are astonishingly good, although they have been shown to be inaccurate for important Gamow-Teller transitions whose strengths are a small fraction of the sum rule limit [12].

\section{Acknowledgments}

We acknowledge the support of the Brazilian funding agencies CAPES and FUJB/UFRJ and by the GSI/Darmstadt. This work has been partially supported by MCT/FINEP/CNPQ(PRONEX) under contract \# 41.96.0886.00. 


\section{REFERENCES}

[1] Proc. of the Int. Symposium on the Physics of Unstable Nuclei, Nucl. Phys. A588, 1 (1995)

[2] C.D. Goodman, C.A. Goulding, M.B. Greenfield, J. Rappaport, D.E. Bainum, C.C. Foster, W.G. Love, and F. Petrovich, Phys. Rev. Lett. 44, 1755 (1980)

[3] T.N. Tadeucci, C.A. Goulding, T.A. Carey, R.C. Byrd, C.D. Goodman, C. Gaarde, J. Larsen, D. Horen, J. Rappaport, and E. Sugarbaker, Nucl. Phys. A469, 125 (1987)

[4] F. Osterfeld, N. Anantaraman, S.M. Austin, J.A. Carr, and J.S. Winfield, Phys. Rev. C45, 2845 (1992)

[5] M. Steiner et al., Phys. Rev. Lett. 76, 26 (1996)

[6] H. Lenske, H.H. Wolter, and H.G. Bohlen, Phys. Rev. Lett. 62, 1457 (1989)

[7] C.A. Bertulani, Nucl. Phys. A554, 493 (1993)

[8] R.D. Lawson, Theory of the Nuclear Shell Model (Clarendon, Oxford, 1980)

[9] G.E. Brown, J. Speth, and J. Wambach, Phys. Rev. Lett. 46, 1057 (1981)

[10] D.E. Greiner, P.J. Lindstrom, H.H. Heckman, B. Cork, and F.S. Bieser, Phys. Rev. Lett. 35, 152 (1975)

[11] M. Buenerd et al., Phys. Rev. C26, 1299 (1982)

[12] S.M. Austin, N. Anantaraman, and W.G. Love, Phys. Rev. Lett. 73, 30 (1994) 


\section{TABLES}

TABLE I. Modified Fermi and Gamow-Teller strengths for single particle transitions and as a function of $q$. The Gamow-Teller and Fermi operators are replaced by their respective product with a spherical Bessel function $j_{0}(q r)$. For $q=0, j_{0}(q r)=1$, and the usual Fermi and Gamow-Teller matrix elements are restored.

\begin{tabular}{|c|c|c|c|}
\hline \hline & $q=0$ & $q=0.5 \mathrm{fm}^{-1}$ & $q=1 \mathrm{fm}^{-1}$ \\
\hline$B_{1 / 2^{-}}(F)$ & 1 & 0.65 & 0.14 \\
\hline$B_{1 / 2^{-}}(G T)$ & 2.66 & 1.73 & 0.37 \\
\hline$B_{3 / 2^{-}}(G T)$ & 0.33 & 0.18 & 0.02 \\
\hline \hline
\end{tabular}

\section{Figure Captions}

Fig. 1 - Differential cross section of the charge exchange reaction ${ }^{13} C\left({ }^{13} N,{ }^{13} C\right){ }^{13} C$ at $105 \mathrm{MeV} /$ nucleon for the double excitation case (target and projectile go to the $3 / 2^{-}$state). Experimental data [5] and calculation are given in arbitrary units.

Fig. 2 - Ratio between the exact calculation and the approximation 0.6 for the charge exchange reaction ${ }^{13} C\left({ }^{13} N,{ }^{13} C\right){ }^{13} C$ at $105 \mathrm{MeV} /$ nucleon, as a function of the scattering angle.

Fig. 3 - Percentage deviation from the exact calculation and the approximation obtained by keeping only the $I=0$ term of the multipole expansion 0.4 for the charge exchange reaction ${ }^{13} C\left({ }^{13} N,{ }^{13} C\right){ }^{13} C$ at $105 \mathrm{MeV} /$ nucleon, as a function of the scattering angle.

Fig. 4 - Ratio between the exact calculation and the approximation 0.6 as a function of an scaling factor $\alpha$ for the charge exchange reaction ${ }^{13} C\left({ }^{13} N,{ }^{13} C\right){ }^{13} C$ at $105 \mathrm{MeV} /$ nucleon. The scaling factor $\alpha$ is used to reduce the pion and the rho masses in the charge exchange interaction. 